\title{
A study of conditions for dislocation nucleation in coarser-than-atomistic scale models
}

\author{
Akanksha Garg, Amit Acharya, Craig E. Maloney \\ Carnegie Mellon University \\ Pittsburgh, PA 15213
}

\begin{abstract}
We perform atomistic simulations of dislocation nucleation in defect free crystals in 2 and 3 dimensions during indentation with circular (2D) or spherical (3D) indenters. The kinematic structure of the theory of Field Dislocation Mechanics (FDM) is shown to allow the identification of a local feature of the atomistic velocity field in these simulations as indicative of dislocation nucleation. It predicts the precise location of the incipient spatially distributed dislocation field, as shown for the cases of the Embedded Atom Method potential for Al and the Lennard-Jones pair potential. We demonstrate the accuracy of this analysis for two crystallographic orientations in 2D and one in 3D. Apart from the accuracy in predicting the location of dislocation nucleation, the FDM based analysis also demonstrates superior performance than existing nucleation criteria in not persisting in time beyond the nucleation event, as well as differentiating between phase boundary/shear band and dislocation nucleation. Our analysis is meant to facilitate the modeling of dislocation nucleation in coarser-thanatomistic scale models of the mechanics of materials.
\end{abstract}

\section{INTRODUCTION}

Homogeneous dislocation nucleation (HDN) has been studied experimentally [1] [2] [3] and through modeling in many papers. Attempts have been made to formulate a nucleation criterion [4] [5] [6] [7] that can be used in larger length-scale analysis to predict the nucleation event. Ideally, the criterion should predict the precise location and instant of instability. It should also be able to predict the line direction and the Burgers vector associated with the nucleating dislocation loop. The simplest attempt to predict nucleation was based on atomic level shear stress, called Schmid stress, which was proven insufficient, when tested through numerical simulations. Rice and co-workers [8] [9] also proposed a nucleation condition for dislocation emission from crack tips, based on the notion of $\gamma$-surface given by Peierls and Nabarro [10] [11], and Vitek [12]. This $\gamma$-surface approach has been shown to be very useful in analysis of nucleation near crack tips [8] [9]. However, this approach fails qualitatively for homogeneous dislocation nucleation [7]. Li et al. [6] introduced the $\Lambda$ criterion, which was based on Hill's analysis [13] of stability of plane waves in a deformed crystal. Miller and Acharya [5] proposed a stress gradient based approach to predict HDN. 
Miller and Rodney (MR) [7] showed that while these criteria work well for certain interatomic potentials and indentation geometries, they fail qualitatively for others. Another fundamental question that was raised by MR is whether the instability is a local or a non-local process. They showed that HDN is inherently a non-local process because of the onset of collective floppiness. Supporting this argument, quantitative estimations of the size of the non-local unstable embryo were obtained in [14]. They also showed that $\Lambda$ predicts the location of incipient nucleation in a diffuse region for HDN irrespective of the interatomic potential and orientation. However, $\Lambda$ cannot predict the instant of nucleation. In this work, we review the previously proposed criteria - the Schmid stress, $\Lambda$ criterion, Stress Gradient criterion and discuss their advantages and limitations. A major inadequancy associated with these criteria is that none of them predicts the instant of nucleation. MR [7] claimed that this is because of their inherently local nature. They also proposed a non-local criterion based on the calculation of eigenvalues of mesoscale atomistic stiffness matrix that precisely predicts the location and instant of nucleation. However, as acknowledged by them, it is not clear how MR's criterion can be extended to larger length scale Discrete Dislocation Dynamics (DD) or continuum analysis. In this work, we propose a technique based on linear stability analysis of the evolution equation for dislocation density in the finite deformation theory of Field Dislocation Mechanics (FDM) [15]-[18]. While we evaluate it based on input from velocity fields calculated from atomistic simulations, the analysis naturally lends itself to application in self-contained continuum analysis including cases of non-homogeneous nucleation. The analysis uses the local velocity gradient field; however it is non-local in the sense that the velocity field is calculated using the boundary conditions, loading conditions and the overall stiffness matrix encapsulating non-local effects.

We validate the predictions of the linear stability analysis of FDM in both two-dimensional and fully three-dimensional simulations. We also examine this technique for different crystal orientations and interatomic potentials such as Embedded Atom Method (EAM) potentials for $\mathrm{Al}$ in addition to simple pair potentials such as Lennard-Jones. This analysis precisely predicts the location and instant of instability. In three-dimensional simulations, the nucleating dislocation density tensor lies in the linear span of critical eigenmodes predicted by the analysis based on FDM. Furthermore, we use a stress gradient criterion [19] to calculate the line direction for 3D simulations. Our results show that the stress gradient criterion predicts the line direction correctly for edge dislocations. However, for mixed dislocations the stress gradient criterion only predicts the edge component of the actual line direction. Interestingly, the proposed analysis discriminates between objective tensor rates; a naturally emergent convected rate succeeds while its substitution by a corresponding rate based on the skew part of the velocity gradient is shown to never predict nucleation.

A very simple overall physical picture emerges for our analysis of nucleation. At the atomistic level, dislocations are simply special arrangements of atoms, not immediately related to deformations of bodies and the compatibility of such deformations. Thus, FDM theory treats them as a special field, separate from a direct connection to the material motion. Special patterns of velocity fields defined on an atomic configuration give rise to the generation of the configurations we call as dislocated. As we explain in detail in this paper, our analysis of dislocation nucleation 
simply constitutes a detailed characterization of instantaneous velocity fields out of any attained configuration of the body (the whole set of atoms involved) that has the potential of generating configurations with dislocations. Interestingly enough, because of the existence of the interatomic spacing between any two atoms, a velocity field defined from discrete atomistic velocities can almost always be considered as continuous, with its gradient being necessarily compatible. It turns out that it is precisely this compatibility of the velocity field that often plays a necessary role in being able to predict the nucleation of a dislocation, classically considered a defect or a lack of compatibility, as shown in sec. IV.

In Sec. II, we describe the modeling details and loading algorithm for different crystal orientations and interatomic potentials. In Sec. III, we review the Schmid stress, the $\Lambda$, and the Stress Gradient criteria. In Sec. IV, we present the FDM linear stability based analysis of dislocation nucleation. In sec. V, we discuss the results of this analysis for different orientations and potentials. Sec. VI contains some concluding remarks.

\section{Simulation Formalism}

\section{A. 2 Dimensional Simulation}

We perform athermal quasi-static nano-indentation simulations for 2-D hexagonal thick crystal films via energy minimization 'dynamics'. The LAMMPS molecular dynamics framework [20] is used to perform simulations. The resulting load-displacement and elastic energy-displacement curves for the indentation process are shown in Fig. 1. The force on the indenter and total elastic energy stored in the crystal increase as the indenter moves into the crystal until the crystal becomes unstable and releases energy. The load drop is accompanied by the nucleation of a dislocation dipole. We are interested in converging to equilibrium configurations just before the nucleation event. To reach as close as numerically possible to the nucleation event, we use a dynamic indenter stepping algorithm similar to the algorithm used by MR [7]. When nucleation occurs, we take an indenter step back, reduce the step size by a factor of ten and restart our simulation.

We set up 2-D hexagonal crystals as shown in Fig. 2 with periodic boundaries on the sides, rigid base at bottom of the crystal, and a circular indenter on top of the crystal. The important geometrical parameters for our setup are: crystalline film width, $L_{x}$; film thickness, $L$; indenter radius, $R$; the crystal orientation with respect to indenter motion axis, $O$. We chose wide $L_{x}$ and thick enough $L$ for each of our setup and verified that increasing the crystalline film width and thickness does not change results significantly. In the rest of the document all length parameters such as $L_{x}, L, R$ and $C$ are measured in units of the lattice constant, $a$.

We use a stiff featureless harmonic repulsive cylindrical indenter for all our simulations. The interaction potential used is of the form:

$$
\begin{aligned}
\phi\left(r_{i}\right)=A\left(R-r_{i}\right)^{2} & \text { if } r_{i} \leq R, \\
0 & \text { if } r_{i}>R .
\end{aligned}
$$

Here $R$ is the radius of the indenter and $r$ is the distance of a particle, $i$, from the indenter center. Similar potentials have been used by other authors [5] [7]. 


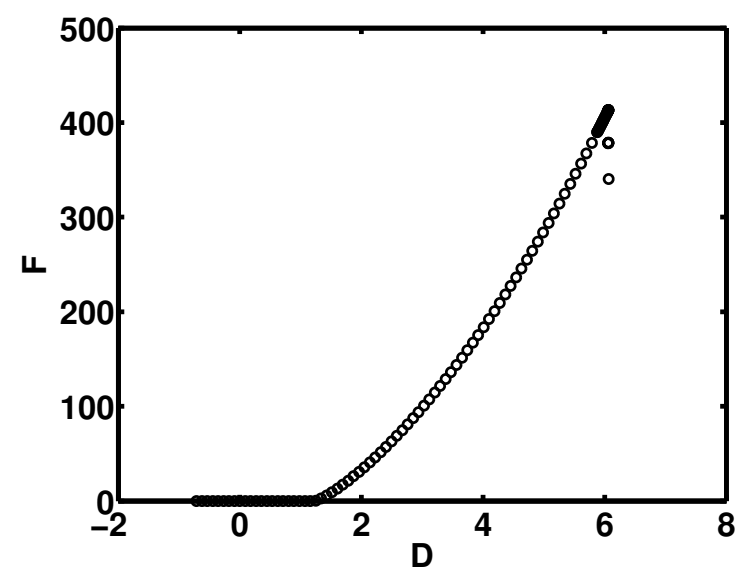

(a)

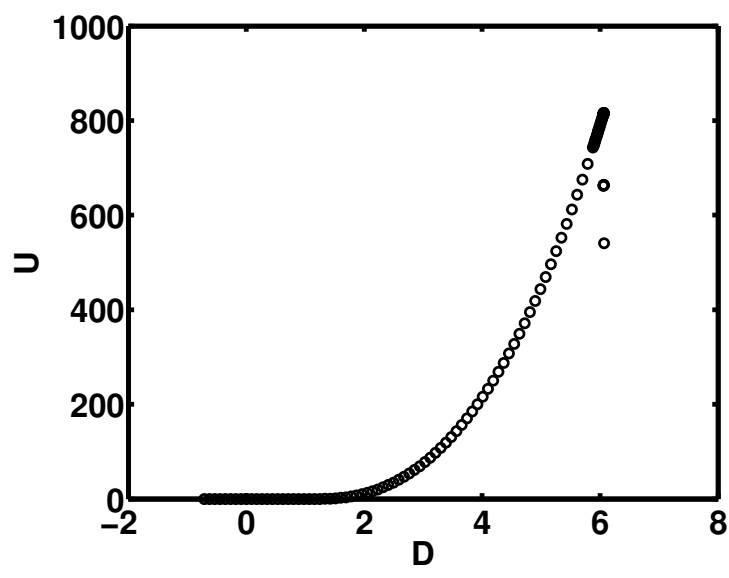

(b)

Fig. 1: (a) Elastic energy, $U$, stored in the crystal as function of indenter depth, $D$, L-J crystal, $L=40, R=40$. (b) Corresponding load, $F$, on the indenter in the vertical direction as function of indenter depth, $D$.

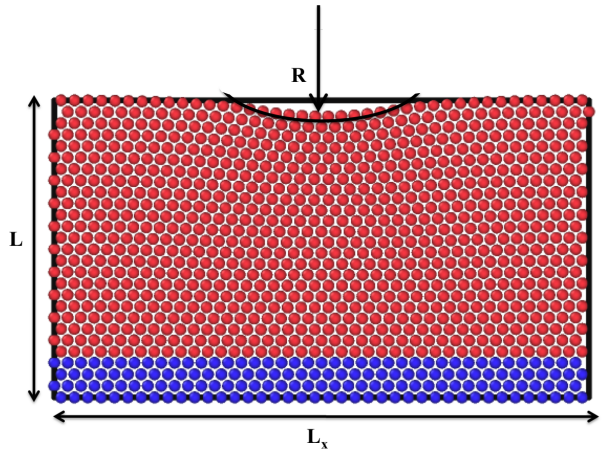

(a)

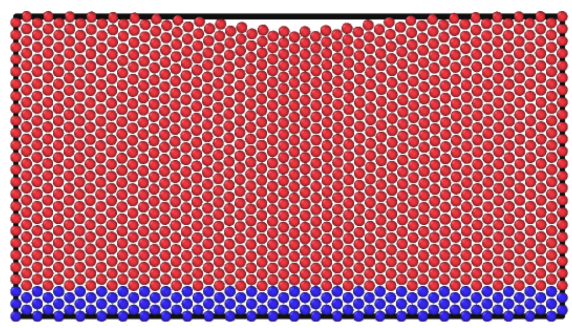

(b)

Fig. 2: Schematic of different orientations of crystal with respect to indenter axis. The red atoms correspond to the crystal. The crystal is periodic in $x$ direction, periodic length given by $L_{x}$. The blue atoms correspond to the rigid base. a) $\mathrm{O}_{1}$ b) $\mathrm{O}_{2}$.

We look at two different orientations: $O_{1}$ and $O_{2}$ for our analysis as shown in Fig. 2. $O_{1}$ has the nearest neighbor axis aligned normal to the indenter motion axis. In $\mathrm{O}_{2}$ the nearest neighbor axis is parallel to indentation direction. $\mathrm{O}_{2}$ is the highest surface energy orientation, hence we had to be careful in avoiding surface defects while indenting these high surface energy orientation systems.

We use the Lennard-Jones (L-J) potential and the EAM potential for Al (Ercolessi Adams [21]). For L-J potential the energies and distances are in L-J units ( $\epsilon$ and $\sigma$ respectively), where 


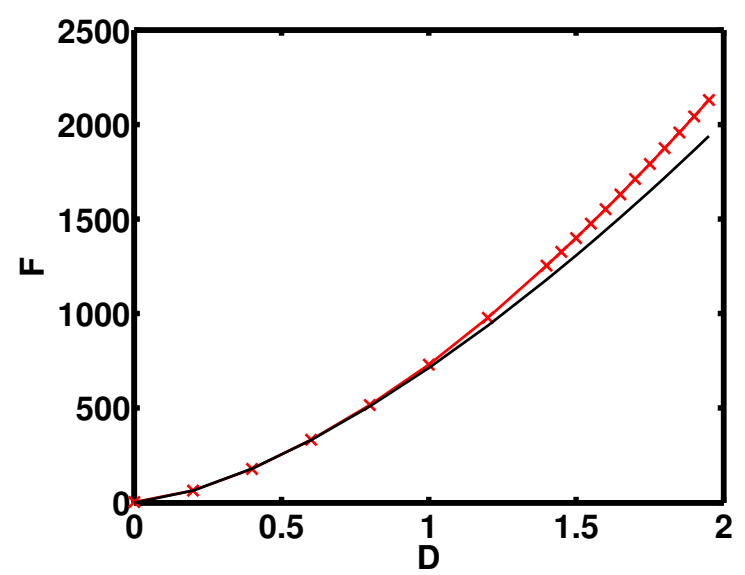

Fig. 3: Indenter Load, $F$, as a function of indenter depth, $D$, for $L=65, R=65$. $F$ vs. $D$ curve from the simulation has been compared to the Hertzian analytical expression $F \propto D^{3 / 2}$.

$\epsilon$ is the well depth of the interatomic potential and $\sigma$ is the distance at which the intermolecular potential between the two particles is zero. For EAM potential, the energies and distances are in $e V$ and Angstroms respectively. All other quantities used in this work such as: force, stress etc. can be expressed in terms of the corresponding length and energy units. The Lennard-Jones (L-J) interaction potential is a pair-potential of the form;

$$
U^{\prime}\left(x_{i j}\right)=\epsilon\left(\left(\sigma / x_{i j}\right)^{12}-\left(\sigma / x_{i j}\right)^{6}\right) .
$$

Here $\epsilon$ and $\sigma$ set the energy and the length scales, and we set them equal to unity. $x_{i j}$ is the distance between particle $i$ and $j$. We also validate the results for both the orientations $O_{1}$ and $\mathrm{O}_{2}$. The total potential energy, $U$, includes interatomic potential energy between particles and interaction energy due to the indentor i.e.

$$
U=\sum_{i j, i \neq j} U^{\prime}\left(x_{i j}\right)+\sum_{i} \phi\left(r_{i}\right)
$$

where $r_{i}$ is $r_{i}\left(x_{i}, D\right)$. Latin characters are used to index particle number, and Greek characters to index Cartesian components. The indenter depth, $D$, represents the indenter motion towards the crystal. At each indenter step, we compute the Hessian matrix, which is the second derivative of the total potential energy with respect to the particle positions,

$$
H_{i \alpha j \beta}=\frac{\partial^{2} U}{\partial x_{i \alpha} \partial x_{j \beta}} .
$$

The first derivative of energy with respect to the particle position gives the force on each particle:

$$
F_{i \alpha}=-\frac{\partial U}{\partial x_{i \alpha}}
$$


$\frac{\partial U(x, D)}{\partial x_{i \alpha}}=0$ represents equilibrium assuming $D$ is an externally prescribed degree of freedom. Then,

$$
\frac{\partial^{2} U}{\partial x_{i \alpha} \partial x_{j \beta}} \frac{d x_{j \beta}}{d t}=-\frac{\partial^{2} U}{\partial x_{i \alpha} \partial D} \frac{d D}{d t} .
$$

The rate of change in forces with respect to the motion of the indenter is denoted by:

$$
\Xi_{i \alpha}=\frac{\partial F_{i \alpha}}{\partial D}=-\frac{\partial^{2} U}{\partial x_{i \alpha} \partial D} .
$$

Since $D$ monotonically increases in time, $t,(6)$ can be written as:

$$
\frac{\partial^{2} U}{\partial x_{i \alpha} \partial x_{j \beta}} \frac{d x_{j \beta}}{d D}=-\frac{\partial^{2} U}{\partial x_{i \alpha} \partial D} .
$$

The forces induced by an infinitesimal external indenter motion must be balanced by the internal atomic rearrangements as shown:

$$
H_{i \alpha j \beta} \dot{x}_{j \beta}=\Xi_{i \alpha}
$$

This is used to calculate particle 'velocities' $\dot{x}_{j \beta}=\frac{d x_{j \beta}}{d D}$. The analytical expression of $H_{i \alpha j \beta}$ can be simply derived for pair potentials such as L-J potential using the following expression (10) from [22],

$$
M_{i \alpha j \beta}=\left(c_{i j}-\frac{t_{i j}}{r_{i j}}\right) n_{i j \alpha} n_{i j \beta}+\frac{t_{i j}}{r_{i j}} \delta_{\alpha \beta} .
$$

where $t$ and $c$ are the first and second derivatives of the bond energy with respect to bond length and $n_{i j \alpha}$ is the unit normal pointing from particle $i$ to particle $j$. Then, $H_{i \alpha j \beta}=-M_{i \alpha j \beta}$ for offdiagonal terms and $H_{i \alpha j \beta}=\sum_{j} M_{i \alpha j \beta}$ for diagonal terms. However, for multibody potentials like the EAM potential the calculation of the Hessian matrix is more involved. For example, there can be non-zero terms in the Hessian matrix for a pair of particles $i$ and $j$ even when $i$ and $j$ are not the neighbors. Thus, the Hessian matrix for a multibody potential like EAM is less sparse than the one corresponding to a pair potential.

The particle velocities computed using this formulation are used subsequently in sec.IV.

\section{B. 3 D Simulation}

We perform computational nano-indentation on a face-centered cubic (FCC) lattice using a spherical indenter of radius $R$. Similar to $2 \mathrm{D}$ simulations, there are periodic boundaries in the perpendicular direction of indenter motion and rigid base at the bottom. The indenter moves along the $\left[\begin{array}{lll}1 & 0 & 0\end{array}\right]$ direction to indent an L-J crystal. The load stepping algorithm is the same as in the two dimensional simulations, Sec. II-A. When nucleation occurs, we take an indenter step back, reduce the step size by a factor of 10 and restart our simulation. The load vs. depth curve for system size, $L=65$ and indenter radius, $R=25$ for fully 3D simulations is shown in Fig. 3. The analytic expression for load vs. indenter depth based on Hertzian contact theory for indentation by parabolic indenter on an anisotropic half space was given by Willis [23]. A spherical indenter can be approximated by a parabolic indenter up to first order. In general for 


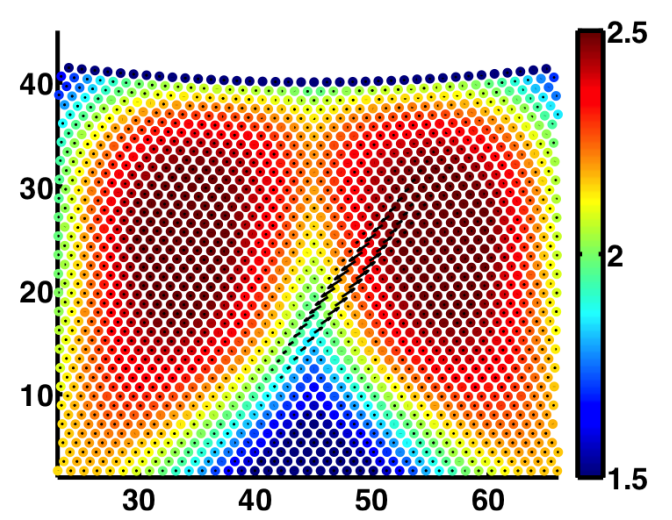

(a)

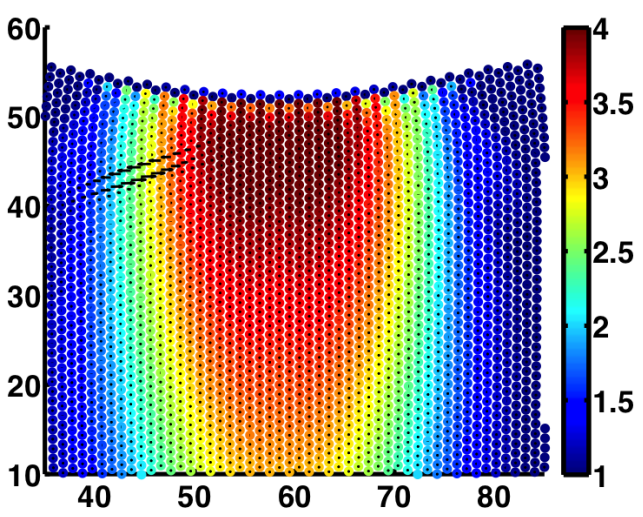

(b)

Fig. 4: The Schmid stress, $\tau$ (given by Eqn. 13) for each atom in L-J crystal just before dislocation nucleation, $O_{1}, R=120$ and $O_{2}, R=50 . \tau$ is not maximum at the point of instability. The arrows at each atom represent velocity field.

an anisotropic half space the contact area is elliptical, however the 4-fold symmetry in the (100) plane results in circular contact area. In this case $F$ vs. $D$ is given by:

$$
F=4 / 3 E^{*} R^{1 / 2} D^{3 / 2} \text {. }
$$

$E^{*}$ is the indentation modulus defined in [23]. As shown in Fig. 3, the analytical Hertzian model fits our simulation results up to the linear regime. The velocity field is computed using (9) as in 2D. However, diagonalization of a Hessian matrix for a fully three dimensional system can be computationally very expensive. The system considered for this work contains approximately 100,000 atoms. At the onset of instability in three dimensional simulations, there are two planes of atoms slipping with respect to each other resulting in nucleation of a dislocation loop. The critical slip plane for an FCC lattice is one (111) plane and the slip direction is $<1 \overline{2} 1\rangle$. Just before nucleation, the slipping plane or the embryo structure is shown in Fig. 12a where the colors represent the magnitude of the velocity field at each atom.

\section{AnAlysis of EXISTING CRITERIA}

\section{A. The Schmid stress}

The simplest attempt to predict the dislocation nucleation process in terms of a single material parameter involves computing appropriate projections of the atomic-level shear stress [24]. Within this framework, it is assumed that a dislocation loop will nucleate when the resolved shear stress, $\tau$, on a given plane exceeds some threshold value, $\tau_{\text {crss }}$. This idea is similar to commonly known local yield stress criteria,

$$
\tau \geq \tau_{\text {crss }}
$$


where,

$$
\tau=\max _{s, n}|s . T . n|
$$

and $T$ is the Cauchy stress tensor. At the embryo, $s$ and $n$ should predict the slip direction and the slip plane normal respectively. It is well established that the existing dislocations become mobile when the resolved shear stress, $\tau$, on a dislocation reaches a critical value, $\tau_{c r s s}$. This idea was extended to predict nucleation. In Fig. 4 the resolved shear stress, $\tau$, just before instability is shown for two different crystal orientations of L-J crystal. The figure shows that $\tau$ is not maximum at the nucleation embryo core and hence, does not predict dislocation nucleation. In many previous studies [6] [25] [26], this idea that the Schmid stress controls dislocation nucleation has been shown to be incorrect.

\section{B. Phonon Stability Criterion $(\Lambda)$}

Li et al. [6] developed a criterion, called $\Lambda$ criterion, based on Hill's [13] analysis of the stability of plane waves in a stressed, elastic continuum. $\Lambda$ is related to the acoustic matrix $A$ defined below which gives the vibrational frequencies of phonons of a given wavevector, $k$, and polarization direction, $p$. It is calculated for a homogeneously deformed crystal with deformation gradient equal to local atomic deformation gradient at $i^{\text {th }}$ particle:

$$
A_{i \mu \nu}(\hat{k})=\lim _{|k| \rightarrow 0} \frac{1}{|k|^{2}} D_{i \mu \nu}(k)
$$

where $|k|:=\sqrt{k_{\alpha} k_{\alpha}}$ is the magnitude of the wavevector $k, A_{i \mu \nu}$ is a $3 \times 3$ matrix indexed by $\mu, \nu$, defined at $i^{\text {th }}$ particle and the dynamical matrix, $D_{i \mu \nu}$, in the long-wavelength approximation can be computed as

$$
D_{i \mu \nu}(k)=|k|^{2} \sum_{j}-0.5 H_{\mu \nu}\left(R_{i j \alpha}\right)\left(\hat{k}_{\beta} R_{i j \beta}\right)^{2}
$$

and $\hat{k}$ represents the unit vector corresponding to the wavevector $k . R_{i j \alpha}$ is the displacement vector defined from the $i^{\text {th }}$ particle to the neighboring particle, $j$, in the homogeneously deformed crystal. $H_{\mu \nu}$ contains the elements of the Hessian matrix for a homogeneously deformed crystal.

$\Lambda$ is defined as the minimum eigenvalue of the acoustic tensor $A$ over all directions $\hat{k}$ on the unit sphere i.e.

$$
\Lambda=\inf _{\hat{k}} \min _{\beta} \operatorname{eig}_{\beta} A(\hat{k}),
$$

where $e i g_{\beta} A(\hat{k})$ represents the $\beta^{\text {th }}$ eigenvalue of the matrix $A(\hat{k})$, and $\beta$ takes values in the set $\{1,2,3\}$.

$\Lambda$ corresponds to the least stable plane-wave perturbation, with $\Lambda=0$ indicating an unstable mode. Alternatively, $\Lambda=0$ may as well be interpreted as the criterion for loss of strong ellipticity of the governing equations of elasticity from a homogeneously deformed state. Thus, one seeks vectors $(p, n)$ s.t. $\left[\mathscr{L}_{r m i q} p_{r} n_{m} p_{i} n_{q}\right]=0$ with $\mathscr{L}$ defined in (23), and the energy function $\psi$ defined as a function of the local atomic deformation gradient based on the Cauchy-Born 


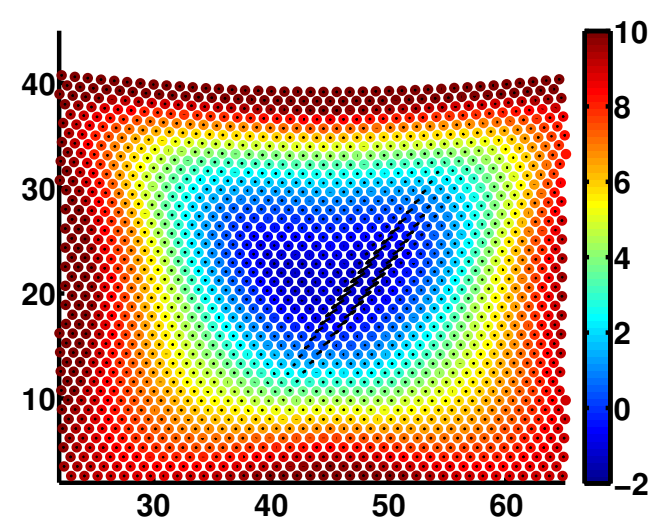

(a)

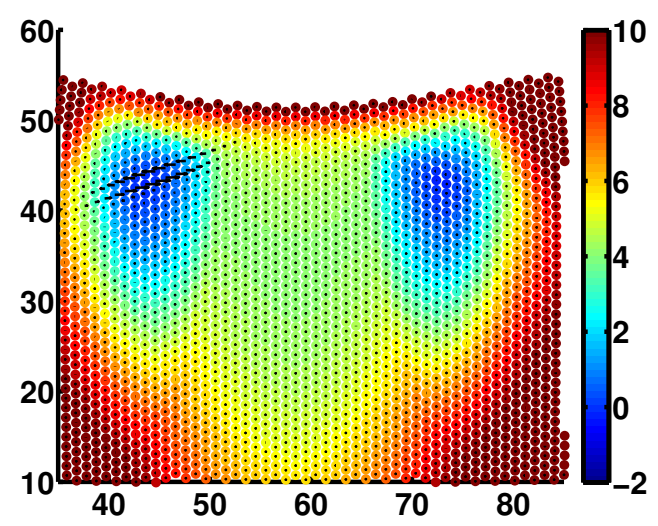

(b)

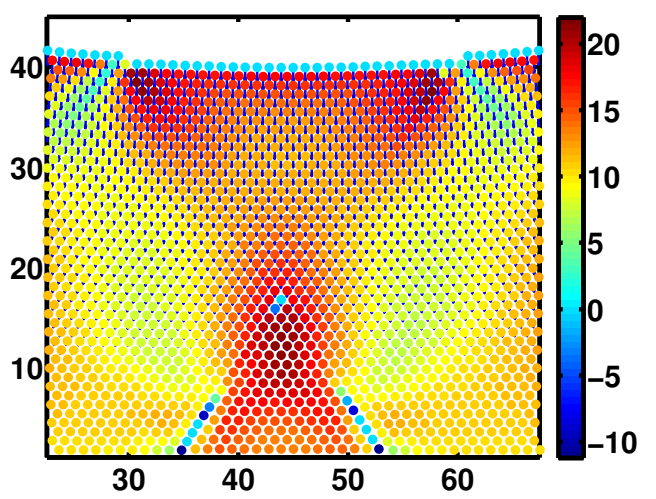

(c)

Fig. 5: Phonon Stability Criterion, $\Lambda$ (given by Eqn. 16) value for each atom in L-J crystal (a) $O_{1}, R=120$, just before nucleation (b) $O_{2}, R=50$, just before nucleation (c) $O_{1}, R=120$ after nucleation. $\Lambda$ is negative in a large region around the dislocation core, so it is difficult to judge the precise location of the core. Moreover, $\Lambda$ decreases further after nucleation when there is no real nucleation in the system.

hypothesis. Recently, MR [7] showed that $\Lambda$ becomes negative before the actual instability and hence, loses the conceptual framework. The detailed calculation of $\Lambda$ is shown in [14]. $\Lambda$ for the L-J potential for the stable surface orientation $O_{1}$ and the unstable surface orientation $O_{2}$ is shown in Fig. 5. In all cases we observe that $\Lambda$ is minimum at the embryo core. Note that $\Lambda$ is also negative at the other symmetrically located embryo core. These results qualitatively remain the same for EAM Al potential as shown in [14]. However, as shown in Fig. 5c, $\Lambda$ decreases further after nucleation around the dislocation cores, when there is no real nucleation in the system. Hence, this criterion cannot be used to predict the nucleation instant. Moreover, $\Lambda$ is negative in a large region around the embryo core and is therefore complicated for identifying 


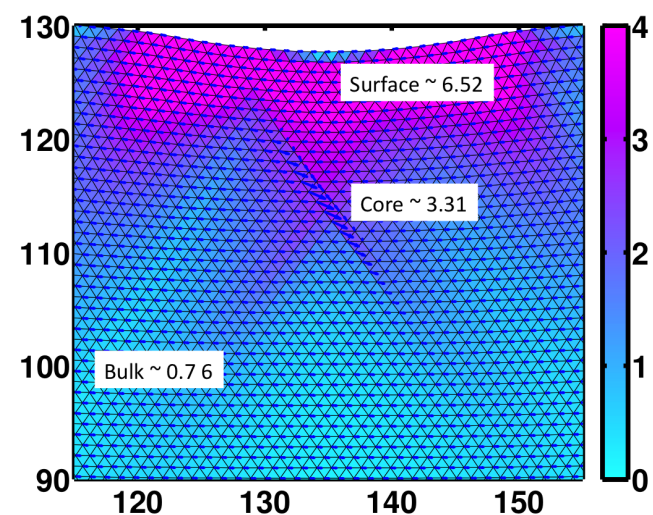

(a)

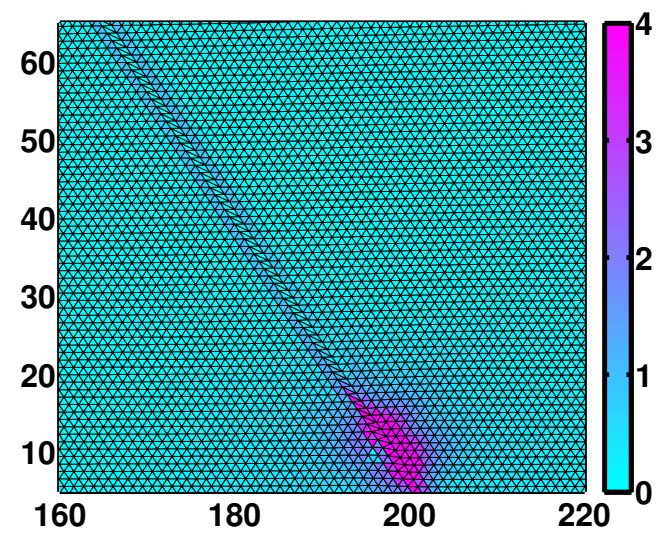

(c)

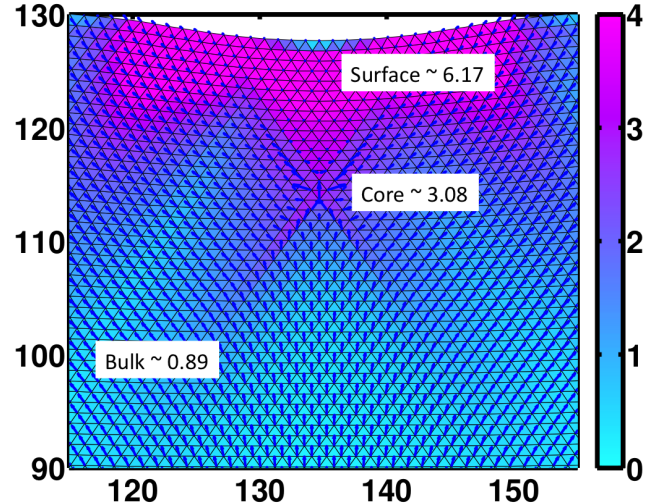

(b)

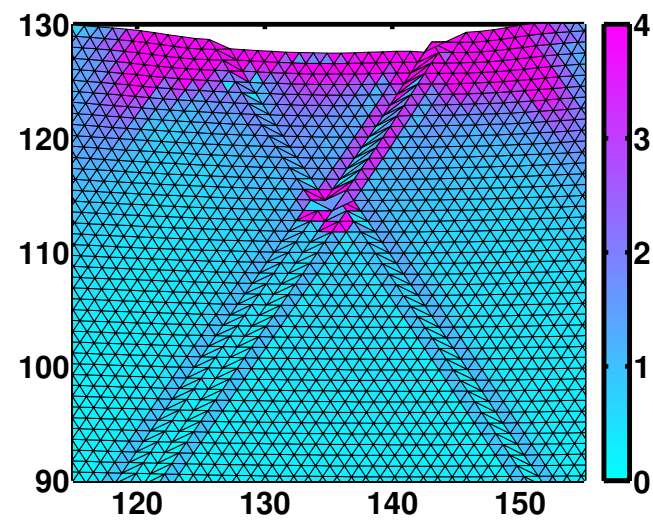

(d)

Fig. 6: Stress Gradient Criterion, maximum $N_{m, l}$ (given by Eqn. 17) value for each atom in L-J crystal, $R=40$, in (a) non-symmetric configuration just before nucleation; (b) symmetric configuration, similar to MA [5] 2004, just before nucleation; (c) non-symmetric configuration, after nucleation; (d) symmetric configuration, after nucleation. Similar to $\Lambda, N_{m, l}$ increases further after nucleation when there is no real nucleation in the system. Also, $N_{m, l}$ is high near the surface so only the bulk has to be considered to calculate the precise location of nucleation, that makes the analysis complex.

the exact location of the core.

\section{Stress Gradient Criterion}

The stress-gradient based criterion [5] identifies the embryo core through a quantity $N_{m, l}$ at each point in the crystal defined as

$$
N_{m, l}=\max _{m, l} \mid \text { m.curlT.l } \mid,
$$


where, $m$ and $l$ are unit vectors in the direction of Burgers vector and line direction respectively for the nucleating dislocation. $T$ is the stress tensor. According to the criterion, if $N_{m, l}$ is greater than a critical value, $N_{c r i t}$, nucleation occurs. $N_{m, l}$ for two different indentation geometries in L-J crystal, $O_{1}$ is shown in Fig. $6 \mathrm{a}$ and $6 \mathrm{~b}$ just before the nucleation. In Fig. 6b, the indenter is symmetrically located with respect to the crystal. This results in nucleation of two symmetrically located embryo loops. The geometry in Fig. 6b is similar to the system in [5], however the interatomic-potential and indenter radius are different. Our results are qualitatively similar to [5], with $N_{m, l}$ high at the core along with the surface. However, the thickness of the high $N_{m, l}$ region at the surface is greater than in [5].

We observed that $N_{m, l}$ is highest at the embryo core in the bulk for all the systems considered. However, similar to $\Lambda, N_{m, l}$ fails to predict the nucleation instant. It increases further near the dislocation cores after nucleation, when there is no actual nucleation in the system. This observation for $\Lambda$ and $N_{m, l}$ was also made by MR [7].

\section{Linear Stability of Dislocation Density Evolution in Field Dislocation MECHANICS}

\section{A. Formulation of Criterion}

We perform linear stability analysis of the equation for evolution of the dislocation density field in finite deformation Field Dislocation Mechanics (FDM). We begin with the evolution equation written in Eulerian form [17]:

$$
\frac{\partial \alpha}{\partial t}=-\operatorname{curl}(\alpha \times(v+V))+s
$$

where the time derivative corresponds to the spatial representation of the $\alpha$ field. Here, $\alpha$ is the dislocation density tensor, $v$ is the material velocity vector, $V$ is the dislocation velocity vector relative to the material and $s$ is a dislocation nucleation rate tensor. A requirement is that $s$ be the curl of a tensor field. The statement (18) arises as the local form of an areal balance statement for Burgers vector content:

$$
\frac{d}{d t} \int_{p(t)} \alpha n d a=\int_{c(t)} \alpha \times V d x+\int_{a(t} s n d a
$$

where $p(t)$ is any oriented area patch of material particles (with unit normal field $n$ ) convecting with the material velocity and $c(t)$ is its closed boundary curve.

With $s=0,(18)$ may also be viewed as a statement of conservation of $\int_{B} \alpha d v$ for any fixed spatial volume $B$ in the absence of any flux of $\alpha$ carried into $B$ by the velocity field $v+V$. However, since $\alpha$ is a 'signed' density, unlike conservation statements for strictly positive scalar density fields like mass, this conservation statement allows nucleation of dislocation density fields within $B$ whose volume integral vanishes, e.g. a single loop contained within $B$. In the spirit of doing more with less, we therefore utilize (18) with $s=0$. Our strategy in this paper involves supplying (18) with a finite-element interpolated (quasi-static) material velocity field from atomistic simulations in which nucleation is monitored, and probing linear 
stability of perturbations to the $\alpha$ field in (18). In addition, our primary interest here is simply in homogeneous nucleation, so we linearize (18) about the state $\alpha=0$. For any physically reasonable constitutive equation for the dislocation velocity $V$, it may be assumed that $V=0$ if $\alpha=0$.

Then,

$$
\frac{\partial \delta \alpha}{\partial t}=-\operatorname{curl}((\delta \alpha \times(v+V))+(\alpha \times \delta v)+(\alpha \times \delta V)),
$$

and since $V=0$ here, the governing equation for linear stability analysis becomes

$$
\frac{\partial \delta \alpha}{\partial t}=-\operatorname{curl}(\delta \alpha \times v)
$$

In terms of components with respect to a rectangular Cartesian coordinate system, (21) can be written as

$$
\frac{\partial \delta \alpha_{i j}}{\partial t}=-e_{j r s}\left(\delta \alpha_{i m} v_{n} e_{s m n}\right)_{, r}
$$

where $e_{j r s}$ is a component of the third-order alternating tensor.

As mentioned before, in our analysis to follow, we utilize a material velocity field obtained from atomistic simulations. For the sake of completeness, we list here the continuum governing equation controlling that velocity field in the special case of an elastic material before any nucleation has happened (including the state for incipient nucleation). Quasi-static balance of linear momentum is the statement

$$
\operatorname{div}[T]=0
$$

where, $T$ is the Cauchy stress tensor and the div operator is on the current configuration. Converted to the statement of continuing equilibrium (or the 'rate form') we obtain

$$
\operatorname{div}\left[\operatorname{div}(v) T+\dot{T}-T L^{T}\right]=0
$$

and for an elastic material with a free-energy density per unit mass given by $\psi(F)$, where $F$ is the deformation gradient from the stress-free elastic reference, continuing equilibrium can be further written as

$$
\operatorname{div}[\mathscr{L} L]=0,
$$

where, $\mathscr{L}_{r m i q}=\rho F_{s m}^{T} \frac{\partial^{2} \psi}{\partial F_{r s} \partial F_{i j}} F_{j q}^{T}$ is the fourth order tensor of incremental moduli, $\rho$ is the mass density, and $L$ is the velocity gradient. Of course, these equations apply to the atomistic material only under the strong assumption that $\psi(F)$ is an adequate representation of the energy density of the crystal.

Henceforth, we use the notation $\delta \alpha=a$. (22) can be rewritten as

$$
\frac{\partial a_{i j}}{\partial t}+a_{i j, r} v_{r}=v_{j, m} a_{i m}-v_{r, r} a_{i j}
$$


In terms of the material time derivative, $(25)$ is equivalent to

$$
\begin{aligned}
\frac{d a_{i j}}{d t} & =K_{i j r m} a_{r m} \\
K_{i j r m} & =\left(v_{j, m} \delta_{i r}-v_{k, k} \delta_{i r} \delta_{j m}\right),
\end{aligned}
$$

and (26) constitutes the governing equation for the perturbation field $a$ of the dislocation density. It is to be noted that (26) represents the vanishing of the (back-leg) contravariant convected derivative [27] of the two-point tensor $a$ with respect to the time-dependent tensor function $\frac{1}{J} F$ with $J=\operatorname{det} F$ and $F$ being measured from an arbitrarily fixed reference configuration:

$$
\operatorname{tr}(L) a+\frac{d a}{d t}-a L^{T}=\frac{d\left(a J F^{-T}\right)}{d t} \frac{1}{J} F^{T}=0 .
$$

With the material velocity considered as a given field, this is simply the linearization of the statement that the convected derivative of the two-point tensor field $\alpha$ vanishes, i.e.

$$
\operatorname{div}(v) \alpha+\frac{d \alpha}{d t}-\alpha L^{T}=0
$$

This is the Lagrangian equivalent of (18) under the assumption that $V=0, s=0$ and div $\alpha=0$ [17], stating that the Burgers vector content of a material area patch remains constant in the absence of dislocation sources and if the existing dislocations threading the patch do not move with respect to the material.

We note that with the velocity gradient field considered as a given input, (26) constitutes a pointwise system of ordinary differential equations (ODE) for the perturbation array $a$. For approximate analysis of stability of this system, we consider it as a constant coefficient system of ODE governed by the velocity gradient field at every point. For the analysis of growth of perturbations it helps to consider the components of $a_{i j}$ as a $9 \times 1$ vector $A$ and $\left(v_{j, m} \delta_{i r}\right.$ $\left.v_{k, k} \delta_{i r} \delta_{j m}\right)$ as a $9 \times 9$ array denoted by $\mathscr{N}$ to write (26) as

$$
\frac{d A}{d t}=\mathscr{N} A \text {. }
$$

If at any stage of deformation an eigenvalue of $\mathscr{N}$ has a positive real part at any point of the body, then that state is deemed to be 'linearly unstable' and susceptible to the nucleation of a dislocation. Since we are dealing with a non-symmetric matrix, there are situations involving eigenvalues with zero real parts with multiplicity greater than one whose modes can show polynomial growth in time, if a linearly independent set of eigenvectors equal to the multiplicity is not available. Our current analysis does not signal potential nucleation in this non-generic case. Of course, linear stability is only conclusive with respect to stability, so for conditions of instability, we treat such positivity as a necessary condition and probe magnitudes of the real parts of the eigenvalues as well.

We denote the maximum of the real parts of the eigenvalues (eigreal part $)$ of $\mathscr{N}$ at any point by the value of the field $\eta$ at that point. The eigen values are computed using eig function in MATLAB based on ARPACK libraries. 


$$
\eta=\max \left(e^{e i g_{\text {real }}} \operatorname{part}(\mathscr{N}(x))\right)
$$

To understand growth of the dislocation density field at the instant of incipient instability, we note that (26) implies

$$
\frac{d\left(a_{i j} a_{i j}\right)}{d t}=2 a_{i j}\left(D_{j m} \delta_{i r}-D_{k k} \delta_{i r} \delta_{j m}\right) a_{r m},
$$

where $D$ is the symmetric part of the velocity gradient $L$, and we observe that our nucleation criterion has the correct limiting behavior in the case of rigid motions, implying that no growth of perturbations in dislocation density (i.e. nucleation) is possible from a dislocation-free state in the case of arbitrary rigid motions. More interestingly, we note the following facts.

\section{B. Convected rate vs. Jaumann rate}

While in our theory the convected derivative with respect to $\left(\frac{1}{J} F\right)$ appearing in (28) is a nonnegotiable ingredient implied by the necessity of doing calculus on a body occupying coherent regions of space parametrized by time, considerations of frame-indifference alone would allow the convected rate to be posed as any appropriate objective rate for the two-point tensor field $\alpha$. In particular, if one were to arbitrarily choose the analog of the Jaumann rate for this two-point tensor field, i.e. the convected rate with respect to the orthogonal tensor $R^{*}$ that at each point of the body satisfies $\frac{d R^{*}}{d t}=\Omega^{*} R^{*}$, where $\Omega^{*}$ is the material spin (the skew-symmetric part of the velocity gradient $L$ ), then (26), (28), and (31) imply that nucleation would never be possible.

\section{Volterra and Somigliana distributions}

Further insight into the possible predictions of nucleation from (26) can be obtained by considering velocity fields with 'planar' spatial variation in only the $x_{1}$ and $x_{2}$ directions and dislocation density perturbations to be constrained to only $a_{i 3} \neq 0$ (i.e. straight dislocations with $x_{3}$ as line direction). Then (25) directly implies

$$
\frac{d a_{i 3}}{d t}=-v_{r, r} a_{i 3} .
$$

In particular, planar simple shearing in the $x_{1}$ direction (only the $v_{1}$ component as non-zero) on planes normal to $x_{2}$ with variation only in the $x_{2}$ direction can cause no nucleation of straight dislocations threading the $x_{1}-x_{2}$ plane (assuming these are the only type of dislocations that are allowed). However, if there exists a slip-direction gradient of the shear strain-rate field, i.e. $v_{1,21}$ is non-zero, then the compatibility of the velocity gradient field (equality of the second partial derivatives) implies that $v_{1,1}$ must be non-zero at such points and that this can cause nucleation of straight edge dislocations according to our criterion (and similarly for the nucleation of straight screws corresponding to shearing in the $x_{3}$ direction with in-plane spatial variations). In particular, if we have an incipient slip embryo where the $v_{1}$ component is uniform in the $x_{1}$ direction except for sharp drop-offs at the boundary of the embryo, then the possibility of a nucleating a Volterra edge dipole exists as shown in Fig. $7 \mathrm{~b}$ and $7 \mathrm{~d}$. On the other hand, if the 


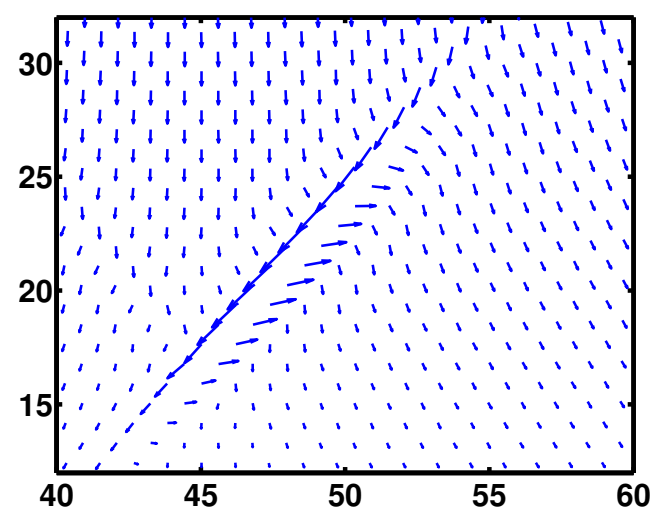

(a)

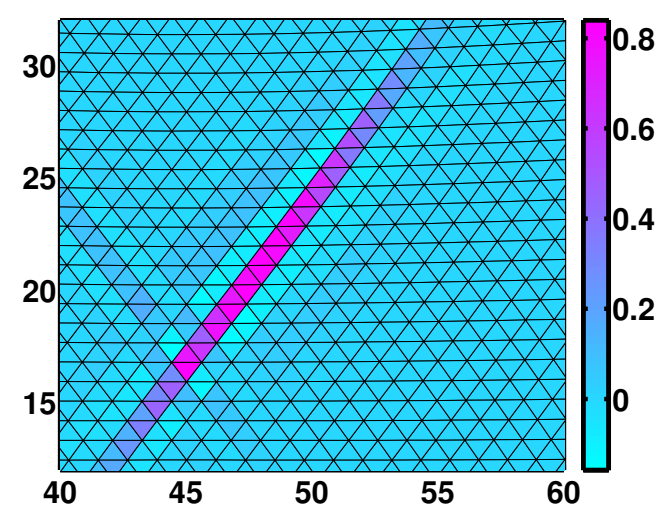

(c)

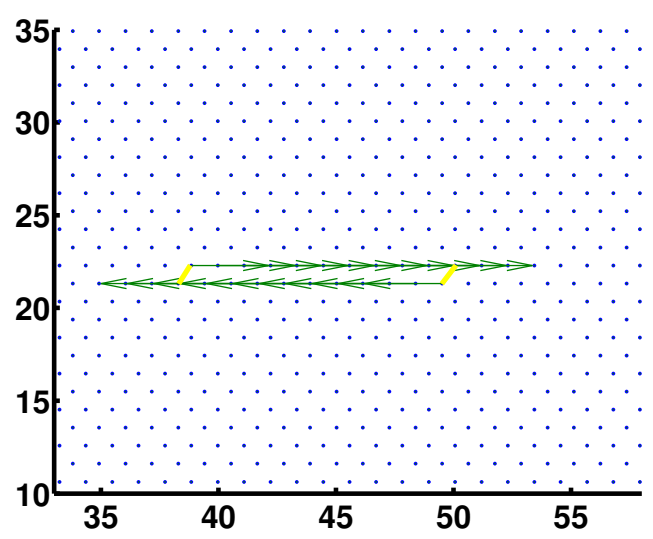

(b)

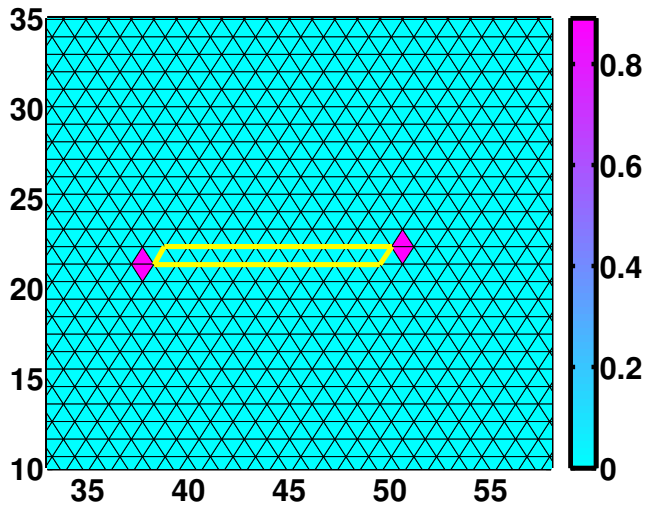

(d)

Fig. 7: L-J crystal, $O_{1}$ (a) Velocity field for $\mathrm{R}=120$, just before nucleation. (b) Idealized velocity field for nucleation of a Volterra dislocation dipole. (c) $\eta$ (given by Eqn. 30) calculated using linear stability of FDM, for velocity field in (a). (d) $\eta$, for velocity field in (b); The yellow-lines in (b) and (d) show the position of the slip embryo. Because of the continuously distributed slip distribution as shown in case (a) $\eta$ is non-local as shown in (c). On the other hand, in (d) $\eta$ is localized at the points of nucleation because of sharp drop-offs in slip at the boundary of embryo in (b).

$v_{1}$ field varies smoothly along the $x_{1}$ direction within the embryo then the possibility nucleating a true continuously distributed dislocation density field exists, corresponding to a Somigliana distribution, as shown in Fig. 7a and 7c. In the simulations of sec. IV, a Somigliana distribution is what appears to nucleate in atomic configurations under load.

Equation (32) was based on the assumption that only $a_{i 3} \neq 0$; however, we note here that Figs. $7 \mathrm{c}$ and $7 \mathrm{~d}$ are plots of the $\eta$ field from calculations that allows for all possible dislocation density perturbations, using the driving velocity fields shown in Figs. $7 \mathrm{a}$ and $7 \mathrm{~b}$, respectively. 


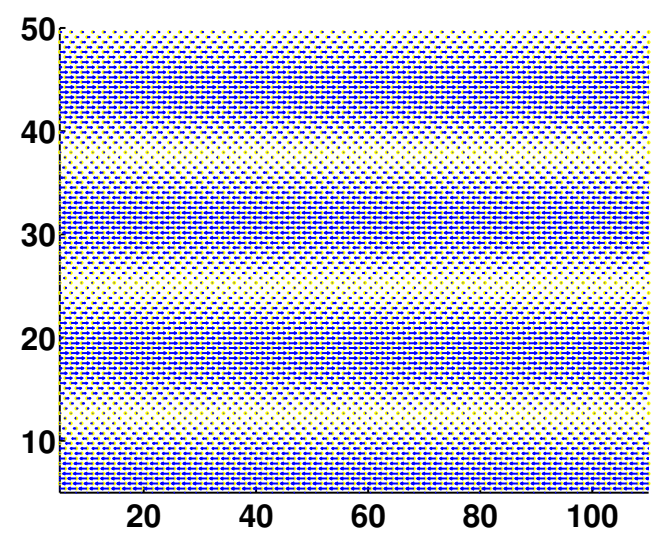

(a)

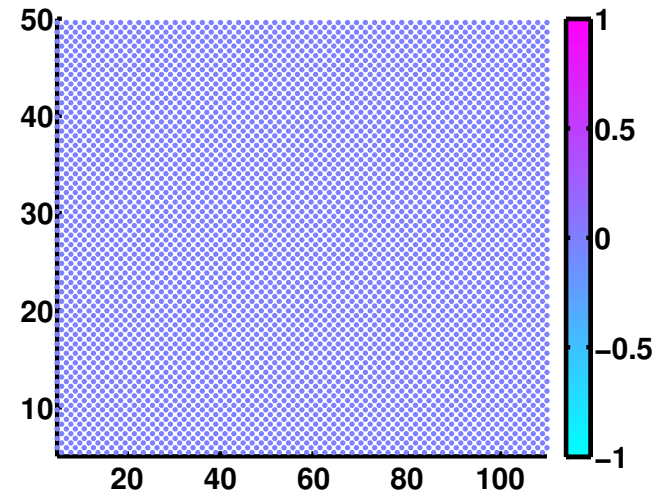

(c)

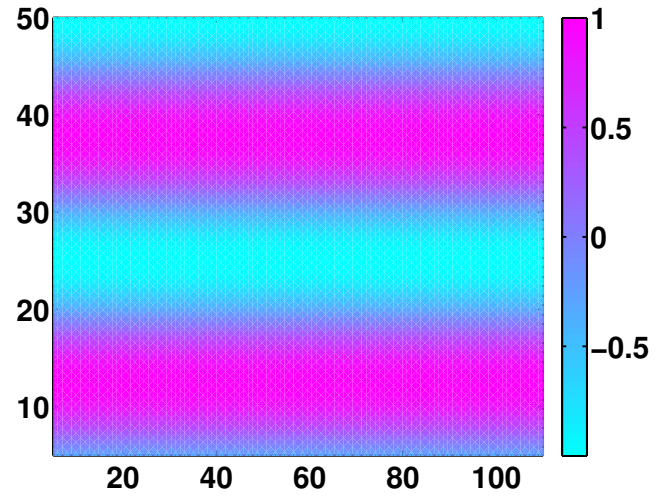

(b)

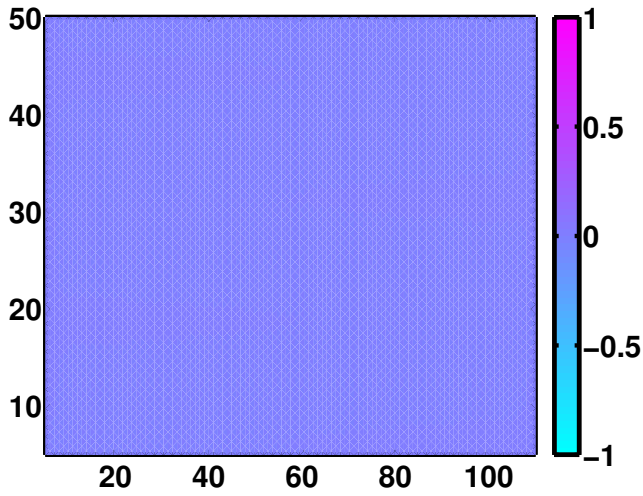

(d)

Fig. 8: L-J crystal, $\mathrm{O}_{2}$ (a) Critical eigenmode for homogeneous compression just before bifurcation. (b) $\Omega / \Omega_{\max }$ (given by Eqn. 33) corresponding to the mode. (c) $\Lambda$ (given by Eqn. 16), $\Lambda$ decreases by an order of magnitude and is almost zero in the whole configuration just before bifurcation. (d) $\eta$ (given by Eqn. 30), $\eta$ is almost zero everywhere and does not show dislocation nucleation. In other words, zero $\Lambda$ shown in (c) implies nucleation everywhere as opposed to $\eta$ shown in (d) that does not show nucleation anywhere.

In sec. V, results utilizing actual atomistic, nanoindentation velocity fields are reported.

\section{Shear band/phase boundary and dislocation nucleation}

The nucleation of a phase boundary or a shear band without terminations within the body are cases that are controlled by the occurrence of localized transverse gradients of the velocity field with respect to some planes. Here, by a phase boundary we mean a single surface in the body across which the deformation gradient is discontinuous; by a shear band we mean two such surfaces separated by a small distance, and 'non-terminating' refers to the fact that these 
discontinuity surfaces run from one external surface of the body to another. Maloney et al. [14] defined $\Omega$ as the transverse derivative of the velocity field with respect to the slip direction to identify location of dislocation nucleation.

$$
\Omega=\hat{n} . \nabla(v . \hat{s}) .
$$

$\hat{s}$ is a direction close to any of the 3 crystal axes passing through a lattice site; it chosen such that it is precisely aligned with a nearest neighbour direction in the current configuration. $\hat{n}$ is the normal to $\hat{s}$. The $\Omega$ field in Fig. 8b is high for the long-wavelength mode shown in Fig. 8a, that is not a case of nucleation of dislocation dipole. Fig. 8 a shows a smooth buckling mode from a state of homogeneous compression. The mode is the linearized precursor of a long-wavelength nonlinear instability that occurs in this simulation with a flat indenter. Note that in the case of homogeneous compression the critical mode is completely non-local and extends to full system size, as compared to the critical mode for nano-indentation discussed in Sec. II-A. In this case of the long-wavelength instability, $\eta$ is close to 0 and does not predict dislocation nucleation as shown in Fig. 8d. In the case of a simple shear where $v_{1,2}$ is non-zero and $v_{1,1}$ is zero, $\eta$ would be zero, whereas $\Omega$ would be high.

For this case of homogeneous compression, $\Lambda$ is almost 0 in the entire configuration as shown in Fig. 8c. Since $\Lambda$ is 0 , it is reasonable to check whether a localized velocity mode with polarization and plane normal predicted using $\Lambda$ is also an eigenmode of the discrete atomistic Hessian matrix, as a check of the adequacy of local continuum elastic response in reflecting the elasticity and instabilities of the atomic lattice. As alluded to in the previous paragraph, we verified that a localized shear band mode predicted by the continuum analysis is not an eigenmode of the atomistic stiffness matrix even though $\Lambda$ is 0 . This difference can be attributed to the atomistic details in the stiffness or the Hessian matrix; roughly speaking, an atomistic model may be assumed to correspond to higher than second-order boundary value problems and the linearization of such a system governing instabilities is naturally different from that of the corresponding second-order system. This analysis suggests that $\Lambda$ cannot always be used even for the case of phase boundary nucleation. Moreover, in this case of homogeneous compression, $\Lambda$ is critical i.e. 0 everywhere and its critical eigenmode does not correspond to the nucleation of a dislocation dipole.

\section{E. Hydrostatic Compression}

In the fully 3-D simulations if a pure hydrostatic velocity field is considered, then

$$
v_{j, m}=e \delta_{j m}
$$

where, $e$ is a constant and (26) becomes

$$
\frac{d a_{i j}}{d t}=-2 e a_{i j}
$$

Since $e$ is negative for compression, $a_{i j}$ always shows growth. As shown in sec. V, our analysis requires $\eta$ should grow by orders of magnitude for implying dislocation nucleation. In this case 
of hydrostatic compression, if the compression rate is uniform then $\eta$ would be constant and would not indicate nucleation. However, by the same token, were a non-uniform-in-time, purely hydrostatic compression state to be achieved in a real deformation, then the FDM based indicator would imply growth of dislocation density.

Leaving aside the question of the physical merit of this case, the reason behind this awkward implication may be understood as follows. From a dislocation-free state, a governing constraint behind the prediction of growth of dislocation perturbations is (28) which is equivalent to

$$
\frac{d}{d t} \int_{p(t)} \alpha n d a=0
$$

for any material area patch $p(t)$ in the body, and the net Burgers vector of any area patch is conserved. Thus, if a deformation tends to shrink areas then the dislocation density has to grow to conserve the Burgers vector content of the perturbation. Interestingly, it appears that it is this kinematic 'mechanism' that predicts correct trends for the initiation of dislocation nucleation as shown in the results of this paper. Of course, once the dislocation density perturbation grows, subsequent states of evolution have non-zero dislocation density and then Burgers vector content of area patches is also affected by the flow term $\alpha \times V$ and its spatial variation.

\section{RESULTS}

We mesh both two and three dimensional systems using Delaunay triangulation. Using sec. II-A, particle velocities are known at each atom or node. We use linear shape functions to interpolate these velocities on each element and compute derivatives. The velocity derivatives are needed to calculate the maximum positive real part of eigenvalues of $\mathscr{N}, \eta$, at the centroid of each triangle (in 2D) or tetrahedron (in 3D). In all figures in this work, the arrows correspond to the particle velocity. In two dimensional simulations, there are two planes of atoms slipping against each other as shown in Fig. 4 and give rise to a pair of dislocations.

For orientation $O_{1}$, the spatial $\eta$ field is shown at various indenter depths for L-J crystal in Fig. 9. $\eta$ is around $6 \times 10^{-4}$ much before nucleation as shown in Figs. 9a and 9b. Note that positive $\eta$ does not necessarily imply nucleation, this being a limitation of constant-coefficient linear stability analysis. Just before nucleation, $\eta$ increases by three orders of magnitude. Also, it is highly positive only for the triangles formed by atoms on the slipping planes. After nucleation, $\eta$ decreases by four orders of magnitude and does not persist at the dislocation cores. In Fig. 10 , we show similar analysis for EAM-Al crystals. Initially $\eta$ is around $6 \times 10^{-9}$ and just before nucleation it increases by three orders of magnitude. Similar to L-J, for the EAM-Al crystal, $\eta$ drops by three orders of magnitude after nucleation. Its interesting that, $\eta$ field in Fig. 9 and Fig. 10 differs by orders of magnitude, even when non-dimensionalized. We believe that this difference is attributed to the different indenter potentials, particulary $A$ in Eqn. 1, used for L-J and EAM potentials. The indenter potential plays a significant role when velocities are computed using Eqn. 9, specifically in $\Xi_{i \alpha}$. Different $A^{\prime} s$ are used to model rigid indenter such that it interacts only with the top layer of atoms and not cause any surface defects. This clearly indicates the need of more realistic ways to model indenter for these simulations. Another important reason begging for more realistic indenters is to benchmark the simulation results against experiments. 


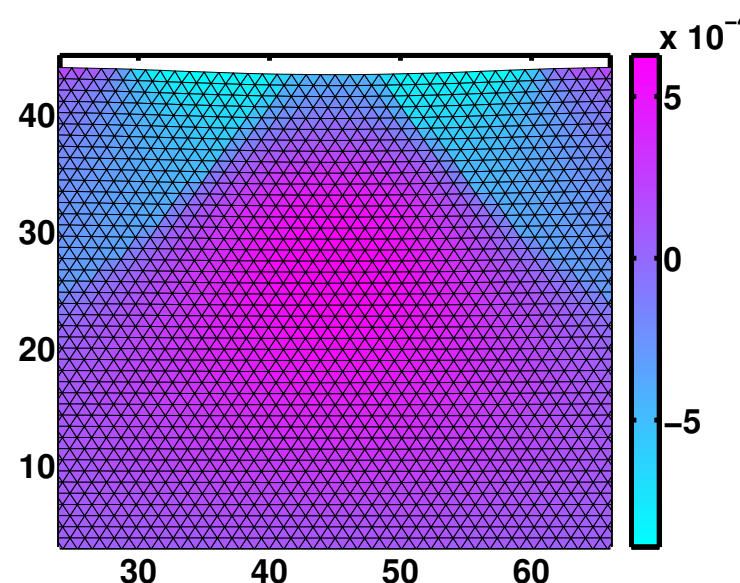

(a)

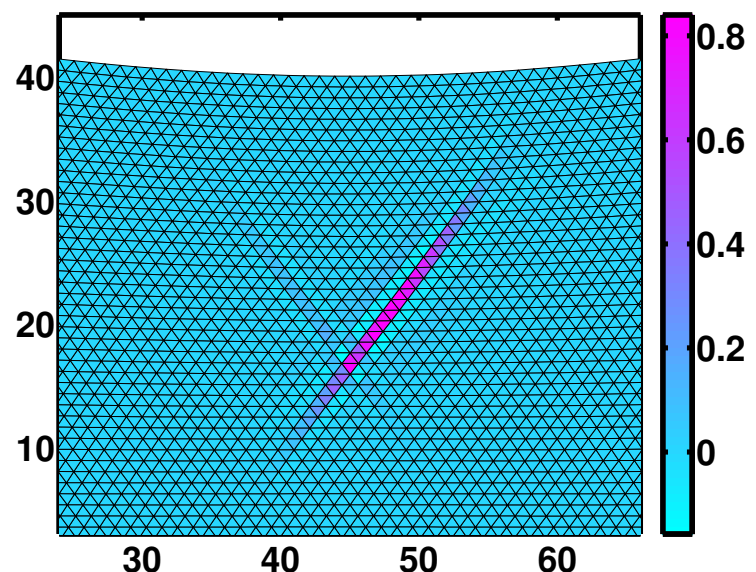

(c)

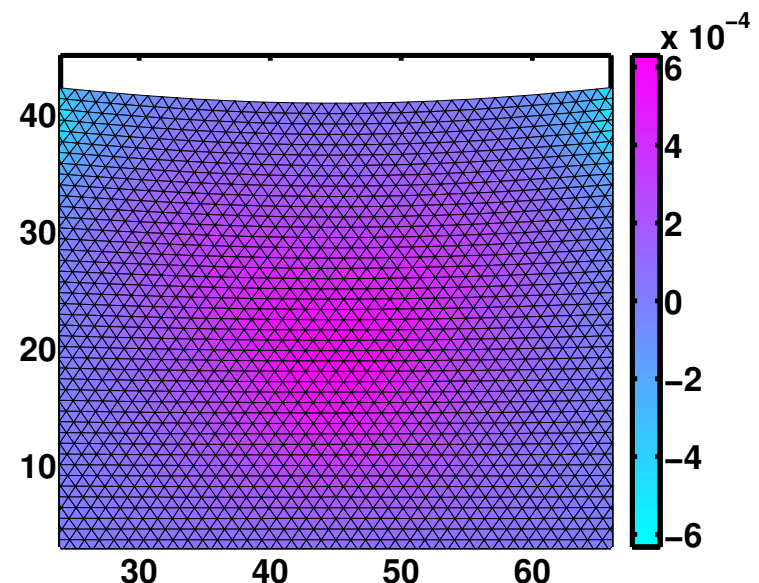

(b)

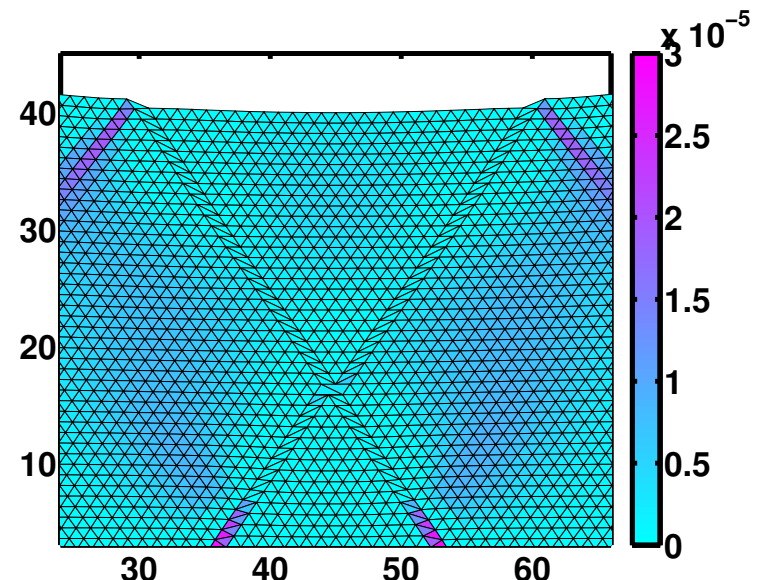

(d)

Fig. 9: $\eta$ (given by Eqn. 30) calculated using linear stability of FDM for L-J crystal, $O_{1}$, $R=120$ in (a),(b) much before dislocation nucleation event; (c) just before nucleation; (d) after nucleation. $\eta$ is precisely maximum at the embryo. It increases by orders of magnitude just before nucleation and decreases by orders of magnitude after nucleation.

Similar results are observed for L-J $\mathrm{O}_{2}$ orientation in Fig. 11. Even though before nucleation $\eta$ depends on the crystal orientation and inter-atomic potential, it increases by three orders of magnitude just before nucleation and decreases by the same amount after nucleation for all systems in $2 \mathrm{D}$.

In Fig. 12a, 12b and 12d, results for the fully 3D simulations are shown. In these figures the FCC lattice is sliced along the plane containing the unstable embryo. In Fig. 12a the colors represent the magnitude of velocity field. Long before nucleation, $\eta$ is around $3 \times 10^{-7}$. Just 


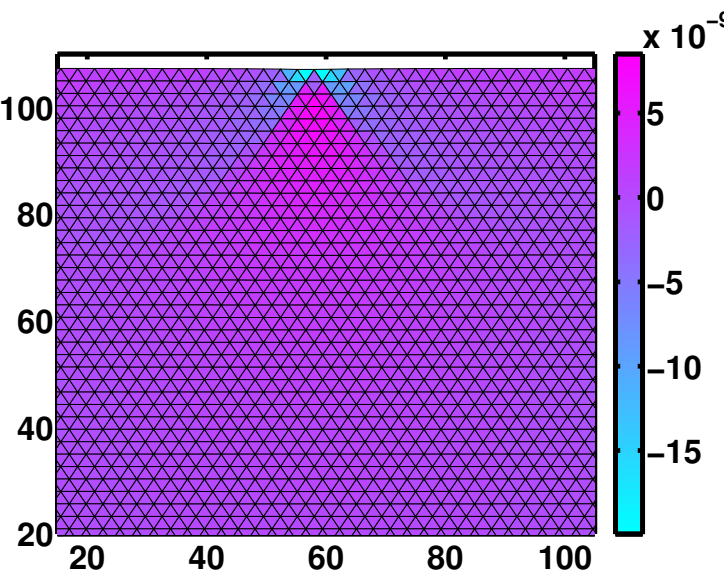

(a)

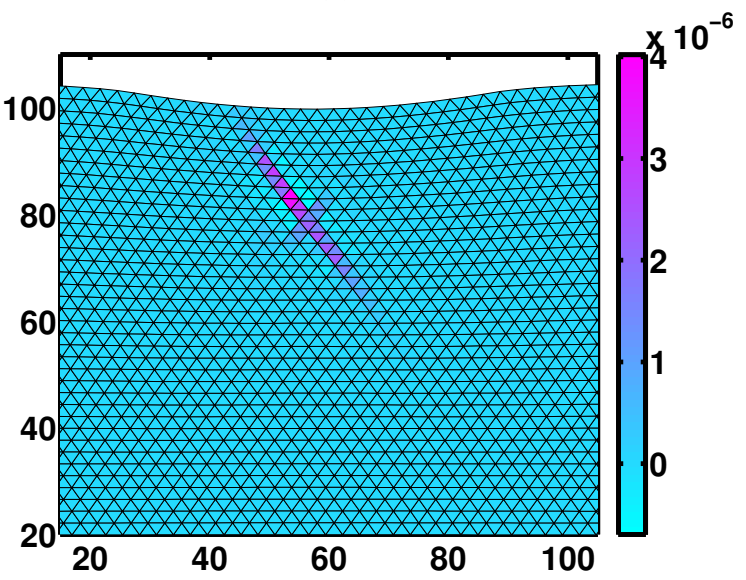

(c)

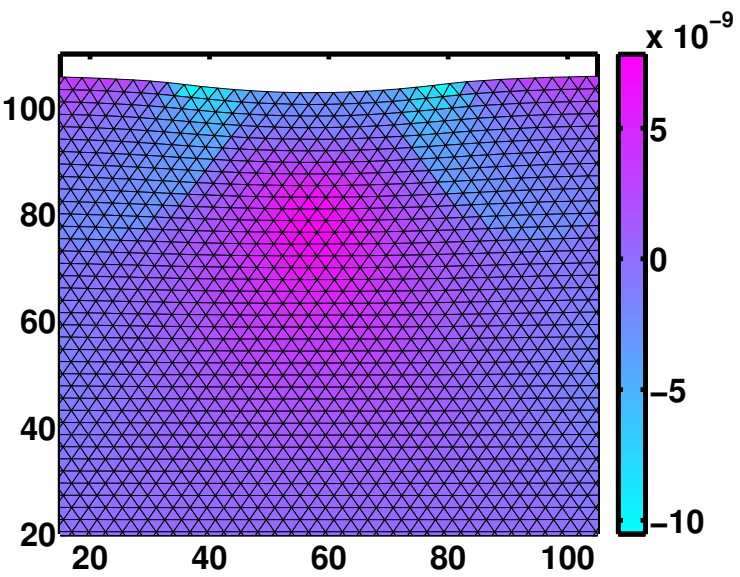

(b)

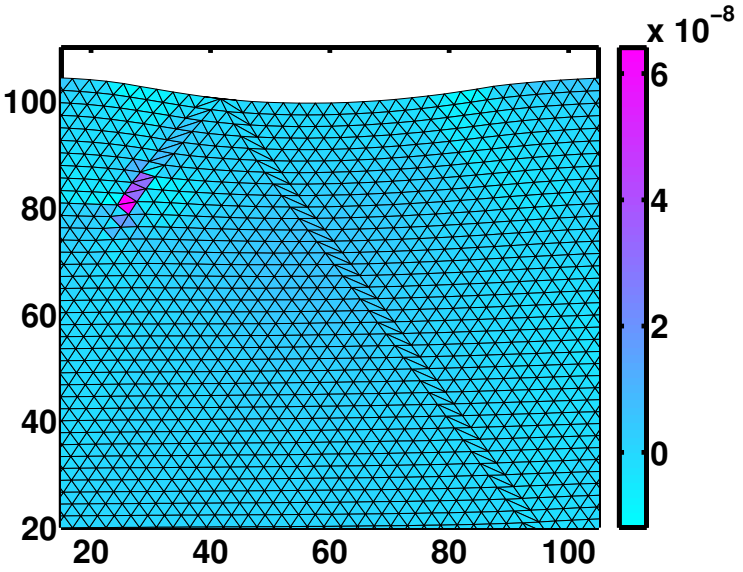

(d)

Fig. 10: $\eta$ (given by Eqn. 30) calculated using linear stability of FDM for EAM-Al. crystal, $O_{1}, R=40$ in (a),(b) much before dislocation nucleation event; (c) just before nucleation; (d) after nucleation. $\eta$ is precisely maximum at the embryo. It increases by orders of magnitude just before nucleation and decreases by orders of magnitude after nucleation.

before nucleation, $\eta$ increases by two order of magnitude as shown in Fig. 12b and then, after nucleation it drops by roughly two orders of magnitude as in Fig. 12d.

In Fig. 13a, the velocity field just before nucleation for an L-J crystal, $O_{1}, R=5$ is shown. Since the indenter is sharp, dislocations nucleate close to the surface. The corresponding $\eta$ field is shown in Fig. 13c. $\eta$ increases by two orders of magnitude before nucleation and successfully predicts nucleation at the surface. The stress gradient criterion couldn't have been used for this case because it is only applicable within the bulk of crystal as discussed in Sec. III-C.

For predicting the line direction, $l$, we calculated the eigenmodes of $\mathscr{N}$. The eigenmodes 


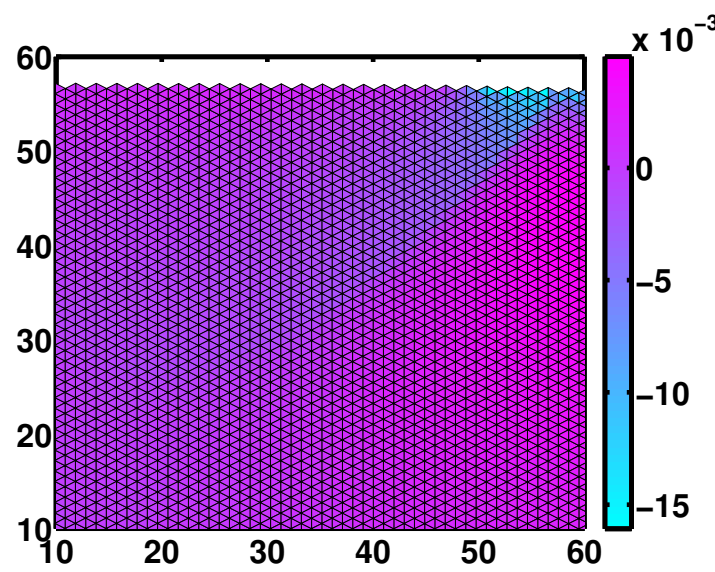

(a)

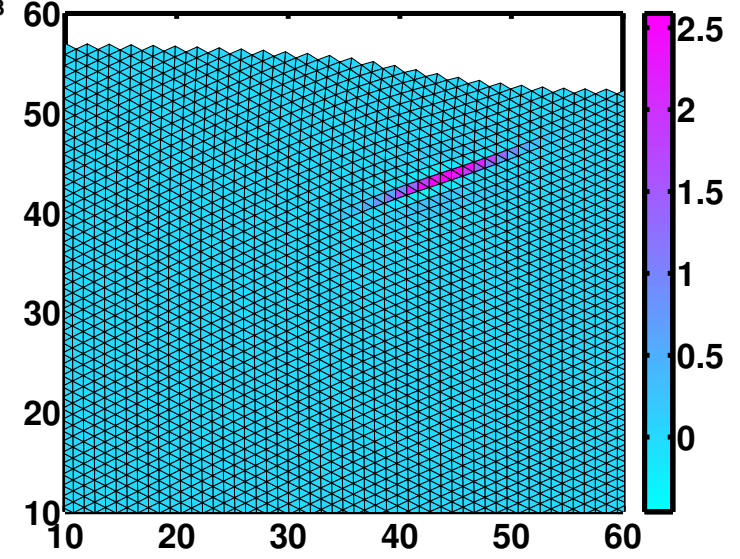

(b)

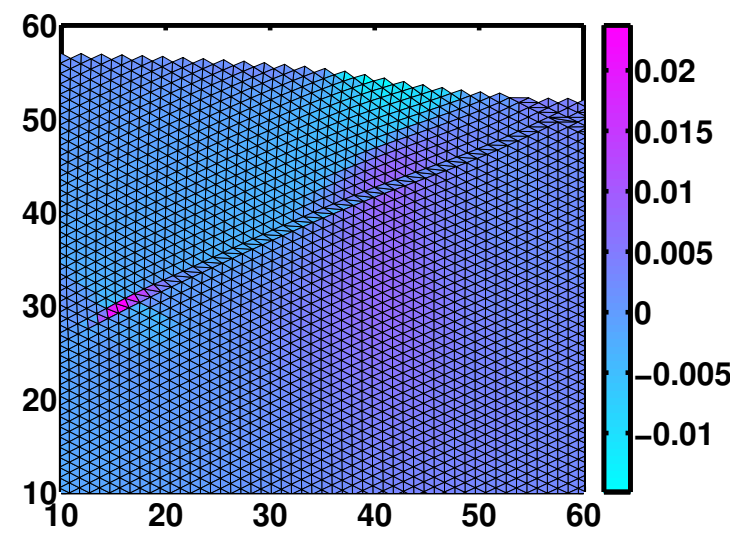

(c)

Fig. 11: $\eta$ (given by Eqn. 30) calculated using linear stability of FDM, for L-J crystal, $\mathrm{O}_{2}$, $R=50$ in (a) much before dislocation nucleation event; (b) just before nucleation; (c) after nucleation. $\eta$ is precisely maximum at the embryo. It decreases by order of magnitudes after nucleation. Only one-half of the crystal in which dislocation nucleation happens is shown.

correpond to the nucleating dislocation density tensor. At the points of interest, $\mathscr{N}$ had more than one eigenvalues with positive real part. We verified that the nucleating dislocation density tensor lies in the linear span of the eigenmodes of the eigenvalues with positive real parts. An expression for predicting the line direction, $l$, was formulated in [19] as shown in (36). This expression is related to the stress gradient criterion described in Sec. III and is given by

$$
l=\nabla \tau \times n .
$$

$\tau$ is the resolved shear stress on the slip plane with normal $n$. We find that (36) predicts the line direction correctly only for edge dislocations, where the line direction is normal to the Burgers 


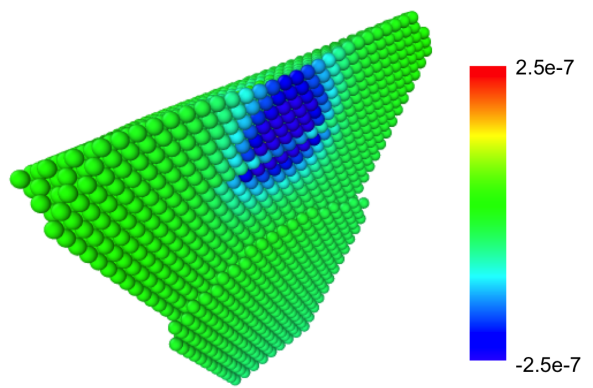

(a)
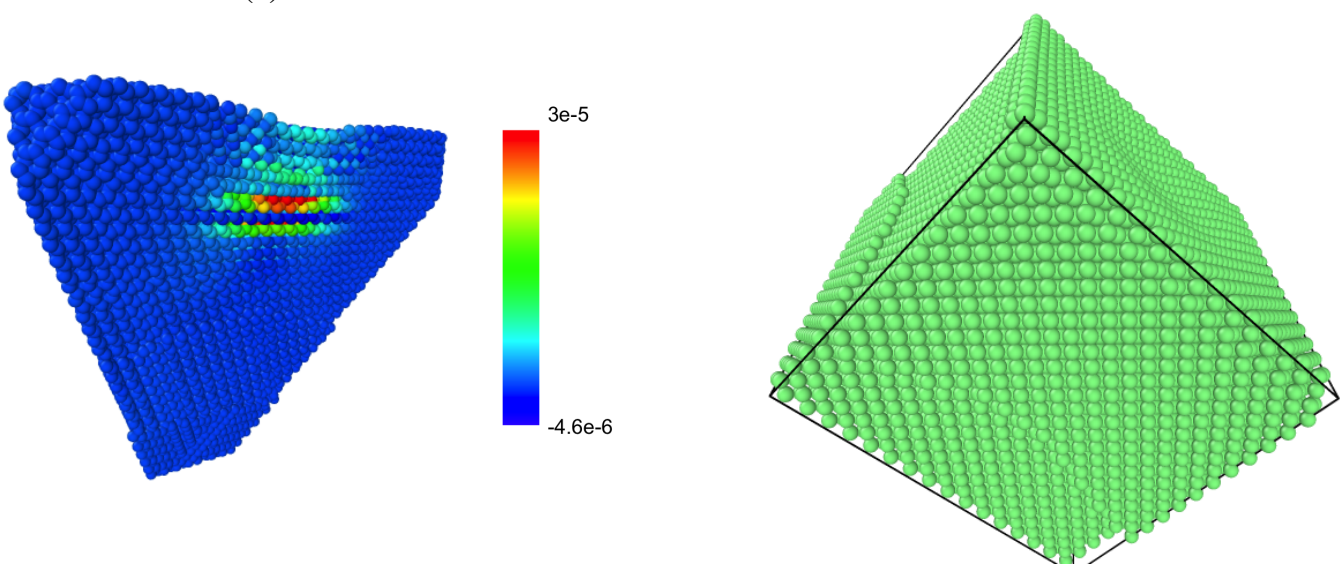

(b)

(c)

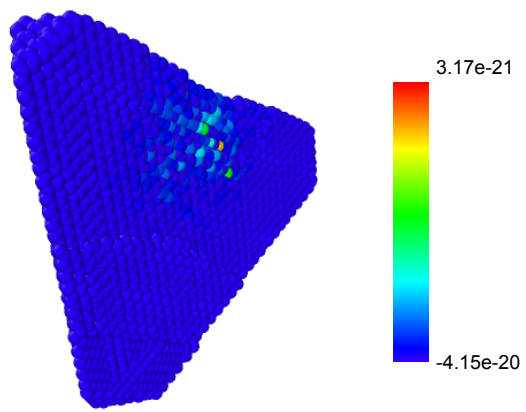

(d)

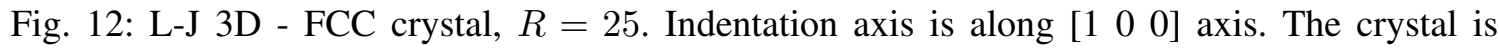
sliced along the plane (111). The colors represent: (a) Velocity field magnitude; (b) $\eta$ (given by Eqn. 30), note that the balls plotted in this figure are located at the centroid of tetrahedrons formed by atoms where $\eta$ is calculated. Similar to 2D, in fully 3D simulations $\eta$ increases by two orders of magnitude just before nucleation and it decreases by two orders of magnitude after nucleation. The full FCC Lattice is shown in (c). There are periodic boundaries conditions in the normal directions to the indentation axis. Black solid lines in (c) show the periodic box size. (d) $\eta$ computed by substituting the Convected rate by Jaumann rates in the linear stability analysis of FDM. Interestingly only the emergent convected rates from linear stability analysis show instability. 


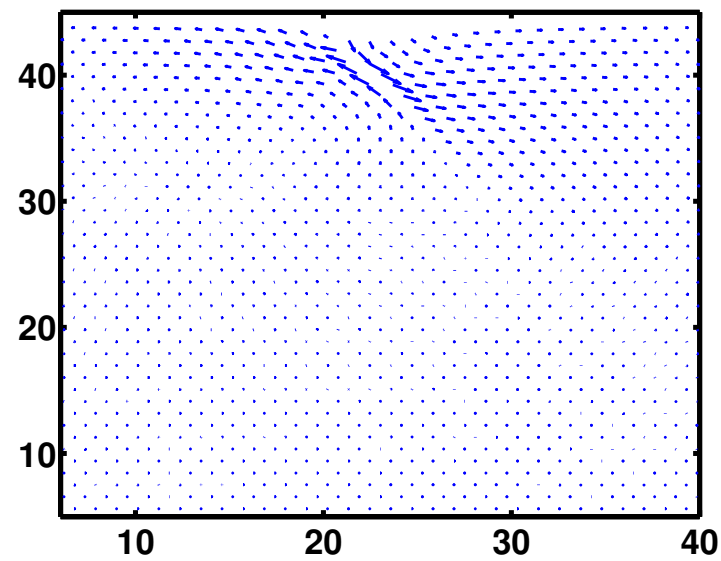

(a)

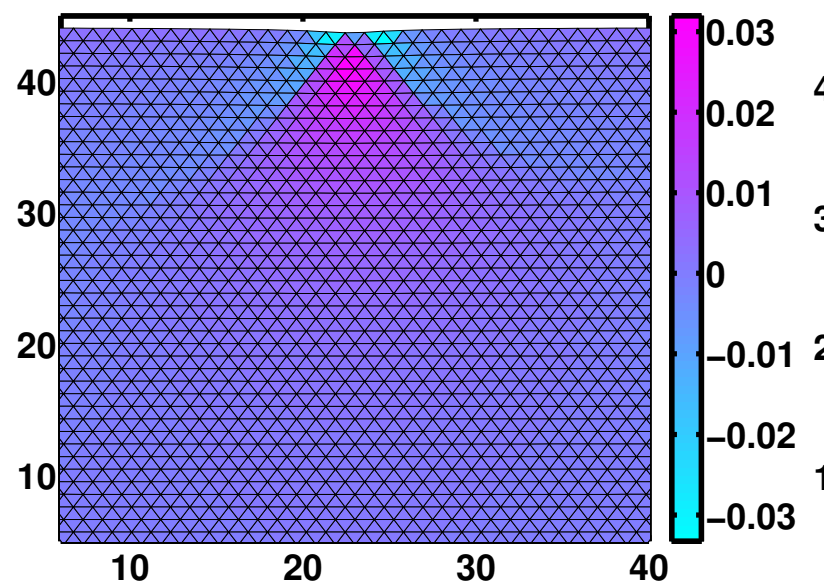

(b)

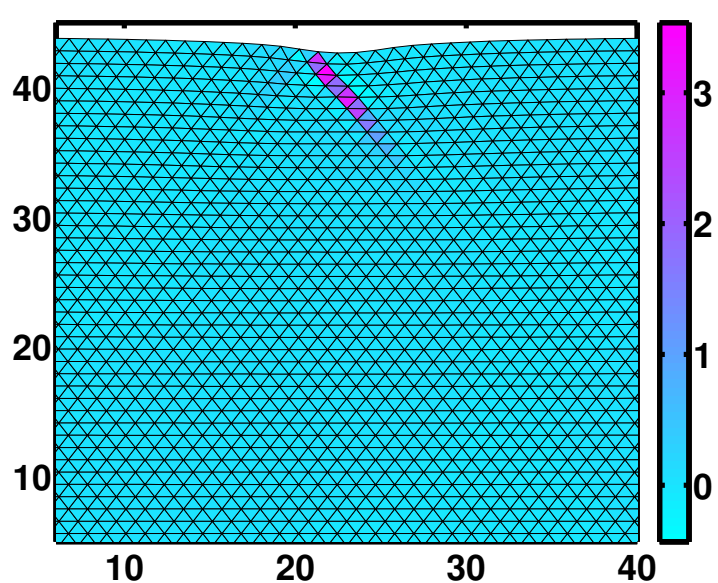

(c)

Fig. 13: Dislocations nucleating very close to surface for L-J crystal, $O_{1}, R=5$ as shown. (a) Velocity field just before nucleation; (b) $\eta$ (given by Eqn. 30) much before dislocation nucleation (c) $\eta$ corresponding to the velocity field shown in (a), just before nucleation.

vector. For mixed dislocations, the stress gradient criterion predicts only the edge component of the actual line direction.

In (25) the convected rate emerges naturally. If we replace the convected rate by the analog of the Jaumann rate for a two-point tensor, $\mathscr{N}$ becomes a skew-symmetric matrix. A skewsymmetric matrix always has imaginary eigenvalues and can never be positive-definite. Hence, the Jaumann rate based $\mathscr{N}$ cannot predict nucleation. The numerical result for maximum eigenvalue of the Jaumann rate based $\mathscr{N}$, just before nucleation, is also shown in Fig. 12d. As the discussion surrounding (31) shows, it does not predict nucleation. 


\section{CONCLUding REMARKS}

An analysis based on the linear stability of field dislocation mechanics (FDM) has been shown to predict dislocation nucleation for several different crystal orientations and inter-atomic potentials. It is observed in Sec. III, that the previous criteria: $\Lambda$ criterion and Stress gradient criterion $\left(N_{m, l}\right)$ cannot predict the nucleation instant. $\eta$, based on the linear stability of FDM, has been shown to predict the nucleation instant in Sec. V. Moreover, even for predicting the location of nucleation the use of previous criteria is not straightforward: $\Lambda$ is negative in a big lobe around the nucleation location and $N_{m, l}$ is high at the surface along with the bulk where nucleation happens, on the other hand $\eta$ is precisely high at the nucleation of location. In Sec. IV, it emerges that $\eta$ is based on very special patterns of velocity field and therefore, can potentially distiniguish between phase boundary and dislocation nucleation; Volterra and Somigliana distributions for nucleation. It can also be used for nucleation at surface as observed in Sec. V. As long as the velocity field has the charecterstics of dislocation nucleation, as observed in this work, $\eta$ should be able to predict nucleation in general (example: nucleation of dislocations at surface or grain boundaries).

The kinematics of dislocation density evolution in FDM appears to be sufficiently versatile in embodying homogeneous dislocation nucleation within the theory and for developing criteria that can be used in other modeling paradigms. In order to isolate and understand this capability, we have tested the feature with atomistically generated velocity fields that, obviously, have analogs in coarser-than-atomic-scale simulation models like Discrete Dislocation Dynamics and Field Dislocation Mechanics. In this sense, our analysis represents an advance in putting forward a conceptual framework for dislocation nucleation that naturally connects to coarser scale models. A main question that arises at this point is the extent to which these coarser length scale models can produce the requisite material velocity fields. Clearly, nonlinear kinematics is important and our analysis in Sec. IV-D shows that dislocation nucleation criteria and associated velocity modes based on classical ideas of loss of strong ellipticity of nonlinear elastic models, even when driven by atomistic input through the Cauchy-Born (CB) hypothesis, may not always be adequate. However, during nano-indentation simulations that induce a strong inhomogeneous deformation, sufficiently close to the bifurcation point of the lattice statics calculation, the polarization direction and discontinuity-plane normal predicted from the (continuum) acoustic tensor corresponding to $\Lambda$ predicts the correct slip plane and Burgers vector direction for the nucleating dislocation dipole. This holds irrespective of the crystallographic orientation and inter-atomic potential. Based on this evidence, dislocation nucleation criteria relying on velocity fields generated from CB-based continuum elasticity coupled with the FDM-based dislocation nucleation indicator we have developed herein appears to be a logical step to pursue in future work. Furthermore, higher-order elasticity can be folded into a framework like Field Dislocation Mechanics and even without resorting to nonlocal/gradient elasticity, FDM in the finite deformation setting incorporating a dislocation density contributing to core energy has a significantly different stress response function [18] than the classical case. The effect of such enhancements in predicted velocity fields from full nonlinear analyses remain to be explored. 


\section{ACKNOWLEDGEMENTS}

This material is based upon work supported by the National Science Foundation under Award Number CMMI-1100245.

\section{REFERENCES}

[1] W. W. Gerberich, J. C. Nelson, E. T. Lilleodden, P. Anderson, and J. T. Wyrobek, "Indentation induced dislocation nucleation: The initial yield point," Acta Materiala, vol. 44, p. 35853598, Sept. 1996.

[2] C. A. Schuh, J. K. Mason, and A. C. Lund, "Quantitative insight into dislocation nucleation from hightemperature nanoindentation experiments," Nature Materials, vol. 4, p. 617621, Aug. 2005.

[3] O. Rodriguez de la Fuente, J. A. Zimmerman, M. A. Gonzalez, J. de la Figuera, J. C. Hamilton, W. W. Pai, and J. M. Rojo, "Dislocation emission around nanoindentations on a (001) fcc metal surface studied by scanning tunneling microscopy and atomistic simulations," Physical Review Letters, vol. 88, p. 036101, Jan. 2002.

[4] K. Van Vliet, J. Li, T. Zhu, S. Yip, and S. Suresh, "Quantifying the early stages of plasticity through nanoscale experiments and simulations," Physical Review B, vol. 67, Mar. 2003.

[5] R. E. Miller and A. Acharya, "A stress-gradient based criterion for dislocation nucleation in crystals," Journal of the Mechanics and Physics of Solids, vol. 52, p. 15071525, 2004.

[6] J. Li, K. J. Van Vliet, T. Zhu, S. Yip, and S. Suresh, "Atomistic mechanisms governing elastic limit and incipient plasticity in crystals," Nature, vol. 418, pp. 307-310, July 2002.

[7] R. Miller and D. Rodney, "On the nonlocal nature of dislocation nucleation during nanoindentation," Journal of the Mechanics and Physics of Solids, vol. 56, pp. 1203-1223, Apr. 2008.

[8] J. R. Rice and G. E. Beltz, "The activation energy for dislocation nucleation at a crack," Journal of the Mechanics and Physics of Solids, vol. 42, pp. 333-360, Feb. 1994.

[9] J. R. Rice, "Dislocation nucleation from a crack tip: An analysis based on the peierls concept," Journal of the Mechanics and Physics of Solids, vol. 40, pp. 239-271, Jan. 1992.

[10] R. Peierls, "The size of a dislocation," Proceedings of the Physical Society, vol. 52, no. 34, 1940.

[11] F. R. N. Nabarro, "Dislocations in a simple cubic lattice," Proceedings of the Physical Society of London, vol. 59, no. 332, p. $256272,1947$.

[12] V. Vitek, "Intrinsic stacking faults in body-centered cubic crystal," Philosphical Magazine, vol. 18, no. 154, p. $773 \&, 1968$.

[13] R. Hill, "Acceleration waves in solids," Journal of the Mechanics and Physics of Solids, vol. 10, pp. 1-16, Jan. 1962.

[14] A. Garg, A. Hasan, and C. Maloney, "Universal scaling laws for homogeneous disocation nucleation during nano-indentation," in preparation.

[15] A. Acharya, "A model of crystal plasticity based on the theory of continuously distributed dislocations," Journal of the Mechanics and Physics of Solids, vol. 49, no. 4, p. 761784, 2001.

[16] A. Acharya, "Constitutive analysis of finite deformation field dislocation mechanics," Journal of the Mechanics and Physics of Solids, vol. 52, no. 2, p. 301316, 2004.

[17] A. Acharya, "Jump condition for GND evolution as a constraint on slip transmission at grain boundaries," Philosphical Magazine, vol. 87, no. 8-9, p. 13491359, 2007.

[18] A. Acharya, "Microcanonical entropy and mesoscale dislocation mechanics and plasticity," Journal of Elasticity, vol. 104, no. 1-2, pp. 23-44, 2011.

[19] A. Acharya, A. Beaudoin, and R. Miller, "New perspectives in plasticity theory: Dislocation nucleation, waves, and partial continuity of plastic strain rate," Mathematics and Mechanics of Solids, vol. 13, no. 3-4, p. 292315, 2008.

[20] S. Plimpton, "Fast parallel algorithms for short-range molecular dynamics," Journal of Computational Physics, vol. 117, pp. 1-19, Mar. 1995.

[21] F. Ercolessi and J. B. Adams, "Interatomic potentials from first-principles calculations," MRS Online Proceedings Library, vol. 291, pp. null-null, 1992.

[22] A. Lemaitre and C. Maloney, "Sum rules for the quasi-static and visco-elastic response of disordered solids at zero temperature," Journal of Statistical Physics, vol. 123, pp. 415-453, Apr. 2006. 
[23] J. R. Willis, "Hertzian contact of anisotropic bodies," Journal of the Mechanics and Physics of Solids, vol. 14, pp. 163-176, May 1966.

[24] R. Phillips, Crystals, Defects and Microstructures: Modeling Across Scales. Cambridge: Cambridge University Press, 2001.

[25] J. Li, T. Zhu, S. Yip, K. J. Van Vliet, and S. Suresh, "Elastic criterion for dislocation nucleation," Materials Science and Engineering: A, vol. 365, pp. 25-30, Jan. 2004.

[26] J. Zimmerman, C. Kelchner, P. Klein, J. Hamilton, and S. Foiles, "Surface step effects on nanoindentation," Physical Review Letters, vol. 87, Oct. 2001.

[27] R. Hill, "Aspects of invariance in solid mechanics," Advances in applied mechanics, vol. 18, pp. 1-75, 1978. 